\title{
Quantum power correction to the Newton law
}

\author{
G.G. Kirilin[ and I.B. Khriplovich] \\ Budker Institute of Nuclear Physics \\ 630090 Novosibirsk, Russia \\ and Novosibirsk University
}

\begin{abstract}
We have found the graviton contribution to the one-loop quantum correction to the Newton law. This correction results in interaction decreasing with distance as $1 / r^{3}$ and is dominated numerically by the graviton contribution. The previous calculations of this contribution to the discussed effect are demonstrated to be incorrect.
\end{abstract}

PACS: 04.60.-m

${ }^{1}$ g_kirilin@mail.ru

${ }^{2}$ khriplovich@inp.nsk.su 


\section{Introduction}

The problem of corrections to the equations of motion, arising in general relativity, is far from being new. The classical relativistic corrections to these equations were found long ago by Einstein, Infeld and Hoffmann [1], and by Eddington and Clark [2]. (A relatively simple derivation of these corrections is presented in the textbook [3].) Later this result was reproduced by Iwasaki by means of Feynman diagrams [4. Thus, the problem of the classical relativistic corrections to the Newton law is solved finally $\mathrm{p}$.

Let us note that the general structure of the relativistic classical correction to the interaction potential of two bodies with masses $m_{1}$ and $m_{2}$, which would be of second order in the Newton gravitational constant $k$, is clear immediately. Indeed, the quantity $k m / c^{2}$ ( $c$ is the velocity of light) has the dimension of length, so that with the account for the symmetry under the interchange $m_{1} \leftrightarrow m_{2}$ the correction should be of the form

$$
U_{c l}=a_{c l} \frac{k^{2} m_{1} m_{2}\left(m_{1}+m_{2}\right)}{c^{2} r^{2}}
$$

The dimensionless constant $a_{c l}$ as found in the above works equals $1 / 2$.

There is one more linear in $k$ combination of constants which can be used for the construction of a power correction to the Newton potential. We mean

$$
\frac{k \hbar}{c^{3}}=l_{p}^{2}
$$

where $\hbar$ is the Planck constant, $l_{p}=1.6 \cdot 10^{-33} \mathrm{~cm}$ is the Planck length. Clearly, such a correction, being of course of quantum nature, should look as follows:

$$
U_{q u}=a_{q u} \frac{k^{2} \hbar m_{1} m_{2}}{c^{3} r^{3}}
$$

One has to find the numerical constant $a_{q u}$. In spite of extreme smallness of the quantum correction, its investigation certainly has a methodological interest: this is a closed calculation of a higher order effect in the nonrenormalizable quantum gravity.

The reason why this problem allows for a closed solution is as follows. The Fourier-transform of $1 / r^{3}$ is

$$
\int d \mathbf{r} \frac{\exp (-i \mathbf{q} \mathbf{r})}{r^{3}}=-2 \pi \ln q^{2}
$$

This singularity in the momentum transfer $\mathbf{q}$ means that the correction discussed can be generated only by diagrams with two massless particles in the $t$-channel. The number of such diagrams of second order in $k$ is finite, and their logarithmic part in $q^{2}$ can be calculated unambiguously.

The corresponding diagrams with photons and massless neutrinos in the loop (see Fig. 1) were calculated by Radkowski [6], Capper, Duff, and Halpern [7], Capper and Duff [8], Duff and Liu [9]. This contribution to the numerical factor $a_{q u}$ is

$$
a_{\gamma \nu}=-\frac{4+N_{\nu}}{15 \pi}
$$

\footnotetext{
${ }^{3}$ Still, erroneous papers on the subject are being published up to now. We mean the articles [5], where the claim is made that the classical relativistic corrections to the equations of motion of two bodies separated by large distance depend essentially on the inner structure of these bodies. We believe that this claim does not withstand criticisms.
} 


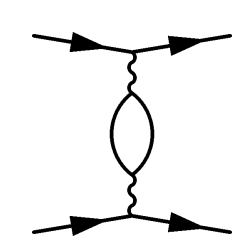

Figure 1: Photon (neutrino) loop

where $N_{\nu}$ is the number of massless two-component neutrinos.

As to the contribution to the effect from the graviton exchange, it was considered by Donoghue [10-13], Muzinich and Vokos [14], Hamber and Liu [15], Akhundov, Belucci and Shiekh [16]. However, there are no quantitative agreement among the results of these works, even the predictions for the sign of the correction differ.

We believe that the correct result for the quantum correction to the Newton law will be sufficiently interesting from the theoretical point of view. This is the aim of our investigation. Among the previous works on the subject, the most detailed presentation of the calculation is given in [11, 16]. Our approach — the direct calulation of Feynman diagrams, the choice of the field operator for the gravitational field and of the gauge — is the same as in $[10-13,16]$. It allows for a relatively detailed comparison of calculations of separate contributions to the effect. This comparison has demonstrated that in $[10-13,16]$ not all diagrams are taken into account, and the considered contributions are calculated incorrectly. Below, when discussing concrete diagrams, we will come back to the comparison with the previous works, including [14, 15. And meanwhile, let us note an obvious error in $[10-13,16]$ : therein formula for the Fourier-transform of the function $1 / r^{3}$ (see (3) ) contains $\pi^{2}$, instead of $\pi$, and this error persists in the final answer as well.

Some of the considered diagrams contribute also to the classical relativistic correction. To check our calculations we computed in parallel these classical contributions and compared them with the corresponding results of [4]. As to these classical corrections, we have complete agreement with [4] for each diagram taken separately.

\section{Propagators and vertices}

We use below the units with $c=1, \hbar=1$.

As a field operator $h_{\mu \nu}$ we choose the deviation of the metrics $g_{\mu \nu}$ from the flat one:

$$
g_{\mu \nu}=\delta_{\mu \nu}+\varkappa h_{\mu \nu} ; \quad \delta_{\mu \nu}=\operatorname{diag}(1,-1,-1,-1) ; \quad \varkappa^{2}=32 \pi k=32 \pi l_{p}^{2} .
$$

We use the gauge where the graviton propagator is

$$
D_{\mu \nu, \alpha \beta}(q)=i \frac{P_{\mu \nu, \alpha \beta}}{q^{2}+i 0} ; \quad P_{\mu \nu, \alpha \beta}=\frac{1}{2}\left(\delta_{\mu \alpha} \delta_{\nu \beta}+\delta_{\nu \alpha} \delta_{\mu \beta}-\delta_{\mu \nu} \delta_{\alpha \beta}\right) .
$$

The tensor $P_{\mu \nu, \alpha \beta}$ is conveniently presented as [17]

$$
P_{\mu \nu, \alpha \beta}=I_{\mu \nu, \alpha \beta}-\frac{1}{2} \delta_{\mu \nu} \delta_{\alpha \beta},
$$

where $I_{\mu \nu, \alpha \beta}=\frac{1}{2}\left(\delta_{\mu \alpha} \delta_{\nu \beta}+\delta_{\nu \alpha} \delta_{\mu \beta}\right)$ is a sort of a unit operator with the property

$$
I_{\mu \nu, \alpha \beta} t_{\alpha \beta}=t_{\mu \nu}
$$




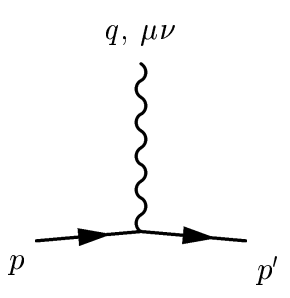

a

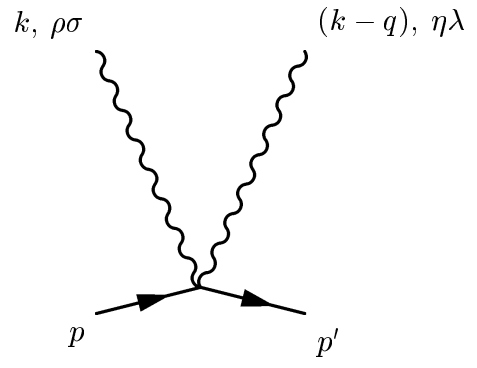

$\mathrm{b}$

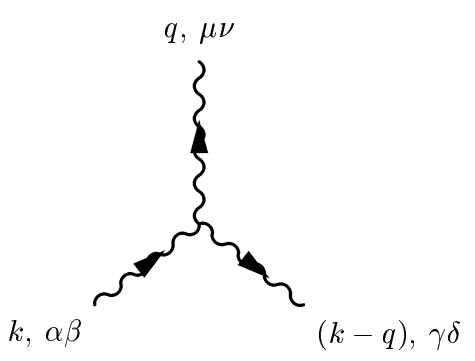

C

Figure 2: Vertices

for any symmetric tensor $t_{\alpha \beta}$. Let us note the following useful identity:

$$
P_{\alpha \beta, \varkappa \lambda} P_{\varkappa \lambda, \gamma \delta}=I_{\alpha \beta, \gamma \delta} .
$$

Propagator of a scalar particle is the usual one:

$$
G(p)=i \frac{1}{p^{2}+i 0}
$$

Vertex of the interaction of a scalar particle with graviton is (see Fig. 2a)

$$
V_{\alpha \beta}\left(p, p^{\prime}\right)=-i \frac{\varkappa}{2}\left[p_{\alpha} p_{\beta}^{\prime}+p_{\alpha}^{\prime} p_{\beta}-\delta_{\alpha \beta}\left(p p^{\prime}-m^{2}\right)\right] \text {. }
$$

The contact interaction of a scalar particle with two gravitons (see Fig. 20b) is

$$
\begin{aligned}
V_{\varkappa \lambda, \rho \sigma}= & i \varkappa^{2}\left[I_{\varkappa \lambda, \alpha \delta} I_{\delta \beta, \rho \sigma}\left(p_{\alpha} p_{\beta}^{\prime}+p_{\alpha}^{\prime} p_{\beta}\right)-\frac{1}{2}\left(\delta_{\varkappa \lambda} I_{\rho \sigma, \alpha \beta}+\delta_{\rho \sigma} I_{\varkappa \lambda, \alpha \beta}\right) p_{\alpha} p_{\beta}^{\prime}\right. \\
& \left.+\frac{\left(p^{\prime}-p\right)^{2}}{4}\left(I_{\varkappa \lambda, \rho \sigma}-\frac{1}{2} \delta_{\varkappa \lambda} \delta_{\rho \sigma}\right)\right] .
\end{aligned}
$$

To our accuracy, one can neglect in this expression the last term, with $\left(p^{\prime}-p\right)^{2}$.

Let us note that in the works [11, [16] the vertex (10) is erroneously presented (and indeed used in the calculations) with a factor two times smaller, $\varkappa^{2} / 2$ instead of $\varkappa^{2}$. We will come back to this factor in Section 4.

The following useful identities are worth mentioning here:

$$
\begin{aligned}
& P_{\mu \nu, \alpha \beta}\left[p_{\alpha} p_{\beta}^{\prime}+p_{\alpha}^{\prime} p_{\beta}-\delta_{\alpha \beta}\left(p p^{\prime}-m^{2}\right)\right]=p_{\alpha} p_{\beta}^{\prime}+p_{\alpha}^{\prime}{ }_{\alpha} p_{\beta}-\delta_{\alpha \beta} m^{2}, \\
& P_{\alpha \beta, \varkappa \lambda} P_{\gamma \delta, \rho \sigma} V_{\varkappa \lambda, \rho \sigma}=V_{\alpha \beta, \gamma \delta} .
\end{aligned}
$$

As to the 3-graviton vertex (see Fig. 2c), which has the most complicated form, we will follow [11] and present it as

$$
\begin{aligned}
V_{\mu \nu, \alpha \beta, \gamma \delta} & =-i \frac{\varkappa}{2} \sum_{i}{ }^{i} v_{\mu \nu, \alpha \beta, \gamma \delta} ; \\
{ }^{1} v_{\mu \nu, \alpha \beta, \gamma \delta} & =P_{\alpha \beta, \gamma \delta}\left[k_{\mu} k_{\nu}+(k-q)_{\mu}(k-q)_{\nu}+q_{\mu} q_{\nu}-\frac{3}{2} \delta_{\mu \nu} q^{2}\right], \\
{ }^{2} v_{\mu \nu, \alpha \beta, \gamma \delta} & =2 q_{\lambda} q_{\sigma}\left[I_{\lambda \sigma, \alpha \beta} I_{\mu \nu, \gamma \delta}+I_{\lambda \sigma, \gamma \delta} I_{\mu \nu, \alpha \beta}-I_{\lambda \mu, \alpha \beta} I_{\sigma \nu, \gamma \delta}-I_{\lambda \nu, \alpha \beta} I_{\sigma \mu, \gamma \delta}\right],
\end{aligned}
$$




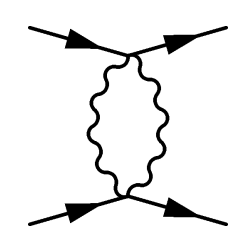

a

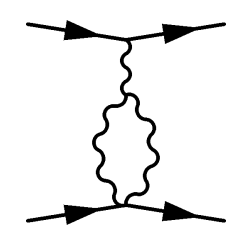

$\mathrm{b}$

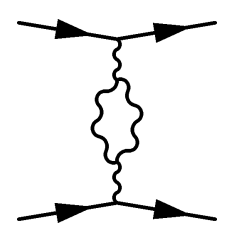

c

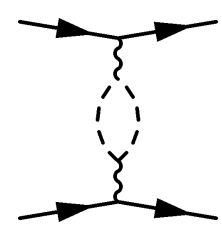

d

Figure 3: Simple loops

$$
\begin{aligned}
{ }^{3} v_{\mu \nu, \alpha \beta, \gamma \delta}= & q_{\lambda} q_{\mu}\left(\delta_{\alpha \beta} I_{\lambda \nu, \gamma \delta}+\delta_{\gamma \delta} I_{\lambda \nu, \alpha \beta}\right)+q_{\lambda} q_{\nu}\left(\delta_{\alpha \beta} I_{\lambda \mu, \gamma \delta}+\delta_{\gamma \delta} I_{\lambda \mu, \alpha \beta}\right) \\
& -q^{2}\left(\delta_{\alpha \beta} I_{\mu \nu, \gamma \delta}+\delta_{\gamma \delta} I_{\mu \nu, \alpha \beta}\right)-\delta_{\mu \nu} q_{\lambda} q_{\sigma}\left(\delta_{\alpha \beta} I_{\gamma \delta, \lambda \sigma}+\delta_{\gamma \delta} I_{\alpha \beta, \lambda \sigma}\right), \\
{ }^{4} v_{\mu \nu, \alpha \beta, \gamma \delta}= & 2 q_{\lambda}\left[I_{\sigma \nu, \alpha \beta} I_{\gamma \delta, \lambda \sigma}(k-q)_{\mu}+I_{\sigma \mu, \alpha \beta} I_{\gamma \delta, \lambda \sigma}(k-q)_{\nu}\right. \\
& \left.-I_{\sigma \nu, \gamma \delta} I_{\alpha \beta, \lambda \sigma} k_{\mu}-I_{\sigma \mu, \gamma \delta} I_{\alpha \beta, \lambda \sigma} k_{\nu}\right] \\
& +q^{2}\left(I_{\sigma \mu, \alpha \beta} I_{\gamma \delta, \sigma \nu}+I_{\sigma \nu, \alpha \beta} I_{\gamma \delta, \sigma \mu}\right)+\delta_{\mu \nu} q_{\lambda} q_{\sigma}\left(I_{\alpha \beta, \lambda \rho} I_{\rho \sigma, \gamma \delta}+I_{\gamma \delta, \lambda \rho} I_{\rho \sigma, \alpha \beta}\right), \\
{ }^{5} v_{\mu \nu, \alpha \beta, \gamma \delta}= & {\left[k^{2}+(k-q)^{2}\right]\left(I_{\sigma \mu, \alpha \beta} I_{\gamma \delta, \sigma \nu}-\frac{1}{2} \delta_{\mu \nu} P_{\alpha \beta, \gamma \delta}\right) } \\
& -k^{2} \delta_{\gamma \delta} I_{\mu \nu, \alpha \beta}-(k-q)^{2} \delta_{\alpha \beta} I_{\mu \nu, \gamma \delta} .
\end{aligned}
$$

In this vertex as well one can neglect, to our accuracy, the last structure ${ }^{5} v_{\mu \nu, \alpha \beta, \gamma \delta}$.

Let us note that in papers [11, 16] vertex (13) is written down with an opposite sign. Our sign is confirmed by the following argument: for physical gravitons with momenta $k$ and $k-q$ vertex (13) should agree in the limit $q \rightarrow 0$ with the interaction (9) of a graviton with a scalar particle.

\section{Simple loops}

It is convenient to start with the diagrams where the Feynman integrals contain two denominators only.

The simplest of them, Fig. 3a, is missed at all in [10-13, 16]. Its calculation causes no difficulty, one has only to use the identity (12) and to go over to the nonrelativistic limit in both 2-graviton vertices. The result for this contribution to the quantum correction is

$$
U_{q u 1}=-\frac{22}{\pi} \frac{k^{2} m_{1} m_{2}}{r^{3}}
$$

The calculation of the next diagram, Fig. 3b, and that obtained from it by interchanging scalar particles, is also sufficiently simple and results in

$$
U_{q u 2}=\frac{26}{3 \pi} \frac{k^{2} m_{1} m_{2}}{r^{3}}
$$

The result of 11] for this contribution differs from (15) only by a wrong power of $\pi$. The corresponding result of [16] is quite different.

As to the diagrams Fig. 3 $3 \mathrm{c}, \mathrm{d}$ with the polarization operator of graviton, we do not have much to add to works [10-13] on the method of the calculation, and nothing to add at all on the result itself (which is used also in [14, 16]). However, for the completeness we present here briefly this calculation. 


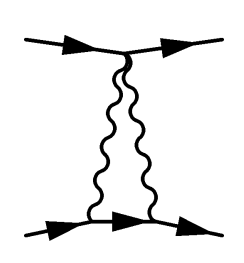

a

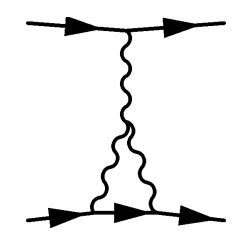

$\mathrm{b}$

Figure 4: Triangle diagrams

The effective Lagrangian corresponding to the sum of these diagrams, with gravitons and vector ghosts, as obtained by 't Hooft and Veltman [17], is

$$
L=-\frac{1}{16 \pi^{2}} \ln \left|q^{2}\right|\left(\frac{1}{120} R^{2}+\frac{7}{20} R_{\mu \nu} R^{\mu \nu}\right) .
$$

To linear approximation the Ricci tensor and scalar curvature of the external gravitational field which enter this expression are

$$
\begin{gathered}
R_{\mu \nu}=\frac{\varkappa}{2} h_{\alpha \beta}\left(q^{2} I_{\mu \nu, \alpha \beta}+q_{\mu} q_{\nu} \delta_{\alpha \beta}-q_{\mu} q_{\alpha} \delta_{\nu \beta}-q_{\nu} q_{\alpha} \delta_{\mu \beta}\right)=\frac{\varkappa}{2} h_{\alpha \beta} r_{\mu \nu, \alpha \beta}, \\
R=\varkappa h_{\alpha \beta}\left(q^{2} \delta_{\alpha \beta}-q_{\alpha} q_{\beta}\right)=\varkappa h_{\alpha \beta} r_{\alpha \beta} .
\end{gathered}
$$

The corresponding contribution to the graviton polarization operator is

$$
\Pi_{\alpha \beta, \gamma \delta}=-\frac{\varkappa^{2}}{8 \pi^{2}} \ln \left|q^{2}\right|\left(\frac{1}{120} r_{\alpha \beta} r_{\gamma \delta}+\frac{7}{80} r_{\mu \nu, \alpha \beta} r_{\mu \nu, \gamma \delta}\right) .
$$

We have taken into account here two possibilities of identifying $R_{\mu \nu}$ and $R$ with the upper and lower external gravitons. The subsequent calculation is straightforward. Let us mention only that the summation over $\mu, \nu$ is conveniently performed at the end. Finally, this contribution to the quantum correction is

$$
U_{q u 3}=-\frac{43}{30 \pi} \frac{k^{2} m_{1} m_{2}}{r^{3}} .
$$

Let us mention that diagrams $3 \mathrm{c}$, d were computed in other variables, $\psi_{\mu \nu}=h_{\mu \nu}-1 / 2 \delta_{\mu \nu} h$, in Refs. [15, 18, 19], and in the source description of gravity due to Schwinger in [6].

\section{Triangle diagrams}

A sort of master formula for triangle diagrams, Figs. đa,b, reads (we keep here only terms singular in $|q|)$ :

$$
i \int \frac{d^{4} k}{(2 \pi)^{4}} \frac{1}{k^{2}(k-q)^{2}\left((p-k)^{2}-m^{2}\right)}=\frac{1}{32 \pi^{2} m^{2}}\left(\frac{\pi^{2} m}{\sqrt{\left|q^{2}\right|}}+\ln \left|q^{2}\right|\right) .
$$

It is conveniently obtained by calculating first the imaginary part of its lhs in the $t$-channel, and then restoring its rhs via the dispersion relation. The first term in the rhs of formula (19) generates $1 / r^{2}$ in the coordinate representation and contributes to the classical relativistic 


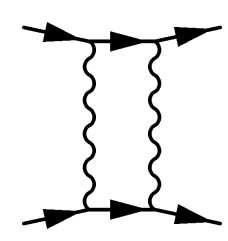

a

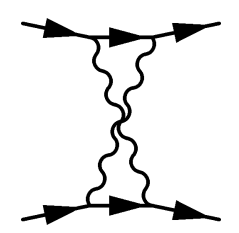

$\mathrm{b}$

Figure 5: Box diagrams

correction. It is kept in (19) to check the calculations by comparison with the corresponding results of 4 .

Our result for the contribution of more simple diagrams of the type Fig. Wa is

$$
U_{q u 4}=\frac{28}{\pi} \frac{k^{2} m_{1} m_{2}}{r^{3}}
$$

This contribution is also missed in [11, 16].

These diagrams contribute to the classical correction as well. An extra proof of our normalization for the seagull vertex is the agreement with the corresponding classical result of [4].

Much more tedious is the calculation of diagrams of the type Fig. 4b. It results in

$$
U_{q u 5}=-\frac{29}{3 \pi} \frac{k^{2} m_{1} m_{2}}{r^{3}}
$$

The corresponding result of [16] differs from (21) only by sign and wrong power of $\pi$. The result of [11] for this contribution is quite different.

\section{Box diagrams}

The expressions for the matrix elements corresponding to the box diagrams Figs. 5a,b can be presented as

$$
\begin{gathered}
M_{s}=i \frac{\varkappa^{4}}{16 m_{1} m_{2}} \int \frac{d^{4} k}{(2 \pi)^{4}} \frac{\left[a-b\left(D_{1}+D_{2}\right)\right]^{2}}{k^{2}(k-q)^{2} D_{1} D_{2}} ; \\
D_{1}=k^{2}-2\left(p_{1} k\right), \quad D_{2}=k^{2}+2 p_{2} k, \quad a=2\left(p_{1} p_{2}\right)^{2}-m^{4}, \quad b=\left(p_{1} p_{2}\right) . \\
M_{u}=i \frac{\varkappa^{4}}{16 m_{1} m_{2}} \int \frac{d^{4} k}{(2 \pi)^{4}} \frac{\left[a^{\prime}+b^{\prime}\left(D_{1}+D_{2}^{\prime}\right)\right]^{2}}{k^{2}(k-q)^{2} D_{1} D_{2}^{\prime}} ; \\
D_{2}^{\prime}=k^{2}-2\left(p_{2}+q, k\right), \quad b^{\prime}=\left(p_{1}, p_{2}+q\right), \quad a^{\prime}=2\left(p_{1}, p_{2}+q\right)^{2}-m^{4} .
\end{gathered}
$$

It is convenient to single out in the numerators of these integrals such structures that cancel one or both denominators $D_{1}, D_{2}\left(D_{2}^{\prime}\right)$. When cancelling a single denominator, one is left with effectively triangle diagrams of the type Fig. 따:

$$
M_{1 s}=i \frac{\varkappa^{4}}{16 m_{1} m_{2}} \int \frac{d^{4} k}{(2 \pi)^{4} k^{2}(k-q)^{2}}\left\{b^{2}\left(\frac{2\left(p_{2} k\right)}{D_{1}}-\frac{2\left(p_{1} k\right)}{D_{2}}\right)-2 a b\left(\frac{1}{D_{1}}+\frac{1}{D_{2}}\right)\right\}
$$




$$
M_{1 u}=i \frac{\varkappa^{4}}{16 m_{1} m_{2}} \int \frac{d^{4} k}{(2 \pi)^{4} k^{2}(k-q)^{2}}\left\{b^{\prime 2}\left(-\frac{2\left(p_{2}^{\prime} k\right)}{D_{1}}-\frac{2\left(p_{1} k\right)}{D_{2}^{\prime}}\right)+2 a^{\prime} b^{\prime}\left(\frac{1}{D_{1}}+\frac{1}{D_{2}^{\prime}}\right)\right\} .
$$

It can be easily demonstrated that to the accuracy we are interested in, expressions (24) and (25) cancel.

Expressions with two denominators deleted,

$$
\begin{aligned}
& M_{2 s}=i \frac{\varkappa^{4}}{16 m_{1} m_{2}} \int \frac{d^{4} k}{(2 \pi)^{4}} \frac{2 b^{2}}{k^{2}(k-q)^{2}}, \\
& M_{2 u}=i \frac{\varkappa^{4}}{16 m_{1} m_{2}} \int \frac{d^{4} k}{(2 \pi)^{4}} \frac{2 b^{\prime 2}}{k^{2}(k-q)^{2}},
\end{aligned}
$$

correspond to the diagrams of the type Fig. Ba. These contributions add up into the following result for the effect discussed:

$$
U_{q u 6}=-\frac{8}{\pi} \frac{k^{2} m_{1} m_{2}}{r^{3}} .
$$

Now we are left with the "irreducible" parts of diagrams Figs. 5ba,b. These irreducible matrix elements are conveniently obtained by calculating first their imaginary parts, in the $s$ and $u$ channels respectively, and then restoring the real parts through the dispersion relations. The results are (we omit nonsingular in $\left|q^{2}\right|$ terms)

$$
\begin{aligned}
M_{0 s} & =i \frac{\varkappa^{4} a^{2}}{16 m_{1} m_{2}} \int \frac{d^{4} k}{(2 \pi)^{4}} \frac{1}{\left(k^{2}-\lambda^{2}\right)\left((k-q)^{2}-\lambda^{2}\right)\left(k^{2}-2 p_{1} k\right)\left(k^{2}+2 p_{2} k\right)} \\
& =-\frac{\varkappa^{4} a^{2}}{\left(16 m_{1} m_{2}\right)^{2}\left|q^{2}\right| \pi^{2}}\left[-1+\frac{s-2\left(m_{1}^{2}+m_{2}^{2}\right)}{6 m_{1} m_{2}}\right] \ln \frac{\left|q^{2}\right|}{\lambda^{2}} ; \\
M_{0 u} & =i \frac{\varkappa^{4} a^{2}}{16 m_{1} m_{2}} \int \frac{d^{4} k}{(2 \pi)^{4}} \frac{1}{\left(k^{2}-\lambda^{2}\right)\left((k-q)^{2}-\lambda^{2}\right)\left(k^{2}-2 p_{1} k\right)\left(k^{2}-2\left(p_{2}+q, k\right)\right.} \\
& =-\frac{\varkappa^{4} a^{2}}{\left(16 m_{1} m_{2}\right)^{2}\left|q^{2}\right| \pi^{2}}\left[1+\frac{u}{6 m_{1} m_{2}}\right] \ln \frac{\left|q^{2}\right|}{\lambda^{2}} .
\end{aligned}
$$

In the above formulae $s=\left(p_{1}+p_{2}\right)^{2}$ and $u=\left(p_{1}-p_{2}-q\right)^{2}$. Expressions (29), (31) are convergent in the ultraviolet sense, but diverge in the infrared limit, depending logarithmically on the "graviton mass" $\lambda$. As usual, such behaviour is directly related to the necessity to cancel the infrared divergence in the Bremsstrahlung diagrams (of course, the gravitational Bremsstrahlung in the present case). Though the leading singularity in $q$ is of the type $\ln \left|q^{2}\right| /\left|q^{2}\right|$, a term with $\ln \left|q^{2}\right|$ arises in the sum of the irreducible boxes as well, and generates the following quantum correction to the Newton potential:

$$
U_{q u 7}=-\frac{23}{3 \pi} \frac{k^{2} m_{1} m_{2}}{r^{3}} .
$$

It is worth mentioning that, as distinct from the previous contributions where $\left|q^{2}\right|$ served as an infrared cut-off for ultraviolet divergent integrals, here $\left|q^{2}\right|$ is the upper limit for infrared divergent integrals.

For the box diagrams as well, we have checked that our results for thus generated classical corrections agree completely with those of $[$ |

The box contributions to the quantum correction are missed at all in [10-13, 16], though diagrams Fig. 5a,b are considered in [20] from another point of view. 
On the other hand, neither in [14], nor in [15] we could find any mention of the "infrared" contribution of the type (33). In fact, in [15] the problem of classical and quantum corrections was treated in different variables, $\psi_{\mu \nu}=h_{\mu \nu}-1 / 2 \delta_{\mu \nu} h$. It can be easily demonstrated that the expressions for the box diagrams are exactly the same in both variables, $\psi$ and $h$. However the box contributions, as calculated in [15], disagree both with the classical ones obtained in [4] (which are demonstrated explicitly in [4] to be the same in both variables, $\psi$ and $h$ ) and with our results for the quantum correction, be it (28), or (33), or the sum of (28) and (33).

At last few words more on Ref. [14]. The approach advocated therein looks quite interesting and promising. However, the results for the quantum correction presented in [14] do not agree with ours (neither do they agree with those of $[10-13,15,16]$ ). Due to the lack of details in [14, we cannot say with certainty what is the origin of the disagreement. Still, an impression arises that at least it is overlooked in [14 that the irreducible triangle diagrams generate not only classical corrections, but quantum corrections as well, i.e. it seems that in [14] the second term is omitted in formula (19).

\section{Conclusions}

Summing up all the contributions obtained, (14), (15), (18), (20), (21), (28), (33), we arrive at the following result for the Newton potential, with the discussed quantum correction due to the two-graviton exchange included:

$$
U(r)=-\frac{k m_{1} m_{2}}{r}\left(1+\frac{121}{10 \pi} \frac{k \hbar}{c^{3} r^{2}}\right) .
$$

Let us note that the derived overall correction enhances, but not suppresses the common Newton attraction.

\section{Acknowledgements}

We are grateful to S. Deser and M.J. Duff for bringing to our attention Refs. [6, 9, 19] and for interesting comments. The investigation was supported in part by the Russian Foundation for Basic Research through Grant No. 01-02-16898, through Grant No. 00-15-96811 for Leading Scientific Schools, by the Ministry of Education Grant No. E00-3.3-148, and by the Federal Program Integration-2002. 


\section{References}

[1] A. Einstein, L. Infeld, B. Hoffmann, Ann. Math. 39, 65 (1938).

[2] A. Eddington, G. Clark, Proc. Roy. Soc. 166, 465 (1938).

[3] L.D. Landau, E.M. Lifshitz, The Classical Theory of Fields, §106, (Pergamon Press, Oxford 1975).

[4] Y. Iwasaki, Progr. Theor. Phys. 46, 1587 (1971).

[5] K.A. Kazakov, Class. Quant. Grav. 18, 1039 (2001); hep-th/0009073;

Nucl. Phys. Proc. Suppl. 104, 232 (2002); hep-th/0107080;

hep-th/0111074;

Class. Quant. Grav. 19, 3017 (2002); hep-th/0201246.

[6] A.F. Radkowski, Ann. of Phys. 56, 319 (1970).

[7] D.M. Capper, M.J. Duff, L. Halpern, Phys. Rev. D10, 461 (1974).

[8] D.M. Capper, M.J. Duff, Nucl. Phys. B44, 146 (1974).

[9] M.J. Duff, J.T. Liu, Phys.Rev.Lett. 85 (2000) 2052;

hep-th/0003237.

[10] J.F. Donoghue, Phys. Rev. Lett. 72, 2996 (1994); gr-qc/9310024.

[11] J.F. Donoghue, Phys. Rev. D50, (1994) 3874; gr-qc/9405057.

[12] J.F. Donoghue, Introduction to the effective field theory description of gravity, in Advanced School on Effective Theories, ed. by F. Cornet and M.J. Herrero (World Scientific, Singapore, 1996); gr-qc/9512024.

[13] J.F. Donoghue, Perturbative dynamics of quantum general relativity, in Proceedings of the Eighth Marcel Grossmann Meeting on General Relativity, ed. by Tsvi Piran and Remo Ruffini (World Scientific, Singapore, 1999); gr-qc/9712070.

[14] I.J. Muzinich, S. Vokos, Phys. Rev. D52, 3472 (1995); hep-th/9501083.

[15] H. Hamber, S. Liu, Phys. Lett. B357, 51 (1995); hep-th/9505182.

[16] A. Akhundov, S. Belucci, A. Shiekh, Phys. Lett. B395, 19 (1998); gr-qc/9611018.

[17] G. 't Hooft, M. Veltman, Ann. Inst. H. Poincare A20, 69 (1974).

[18] D.M. Capper, G. Leibbrandt, M. Ramon Medrano, Phys. Rev. D8, 4320 (1973).

[19] M.J. Duff, Phys. Rev. D9, 1837 (1973).

[20] J.F. Donoghue, T. Torma, Phys. Rev. D54, 4963 (1996); hep-th/9602121. 